\title{
INTELLIGENT SUPPLY CHAIN PLANNING IN VIRTUAL ENTERPRISES
}

\author{
Dmitry A. Ivanov \\ State research center of Russia, \\ Central Research Institute of Robotics and Technical Cybernetics \\ 194064 Saint Petersburg, RUSSIA \\ dmitri.ivanov@mail.ru \\ Alexander V. Arkhipov \\ State research center of Russia, \\ Central Research Institute of Robotics and Technical Cybernetics \\ 194064 Saint Petersburg, RUSSIA \\ a_arkhipov@ipi.neva.ru \\ Boris N. Sokolov \\ Russian academy of science, \\ Saint Petersburg Institute of Informatics and Automation \\ 199178 Saint Petersburg, RUSSIA \\ sokol@iias.spb.su
}

\begin{abstract}
This paper addresses the methodological problem of dynamical supply chain planning in virtual enterprises. The rapid evolution into intelligent supply chains characterized by flexible structuring and dynamical interactions poses a problem of novel planning concepts and models. Our main research focus is elaboration of methodological and technical basis of supply chain analyzing, modeling, structuring and scheduling on the basis of the multi-agent approach as conceptual Integrated planning framework. For its realization we elaborated an agent model and functional architecture of the multi-agent system for intelligent support of collaborative processes in virtual enterprises. A general formulation of dynamic supply chain planning is given.
\end{abstract}

\section{INTRODUCTION}

In our approach, a virtual enterprise (VE) is a dynamic business-system, which contains an aggregate of amalgamated technological resources (competences) of autonomous business units (agents) within a common information (virtual) space on the temporary cooperative basis. These units are able to produce products or services through their co-operation and operative resources distribution (Ivanov, 2003). The vertical and horizontal customer oriented networking leads to new problems for the supply chain management (SCM) (Kaihara, 1999). The traditional supply chains, which have stable structures and determined interactions, evolve into intelligent 
supply chains characterized by flexible structuring and dynamical interactions (Zeng, Sycara, 1999, Swaminathan et al., 1998). The special feature of the SCM in VE consists in flexibly configurable supply chains, conditioned by an enlargement of alternatives to search suitable partners for the cooperation. That gains complexity because the manufacture of a product might be carried through via several variants and several agents can again complete partial performances within those variants.

In our approach, supply chain planning is a macro-function, which contains supply chains dynamical analyzing, modeling, structuring and scheduling. Three problems of supply chain planning are to solve using a common methodological framework (s. figure 1):

- Synthesis of the VE's structure (building of a competence pool in accordance with the technological details of the product),

- Synthesis of the supply chain structure (building of the supply chain through partner selection of this pool in accordance with the economical and administrative information, e.g. due dates, priorities, costs, etc.) and

- Scheduling in the supply chain.

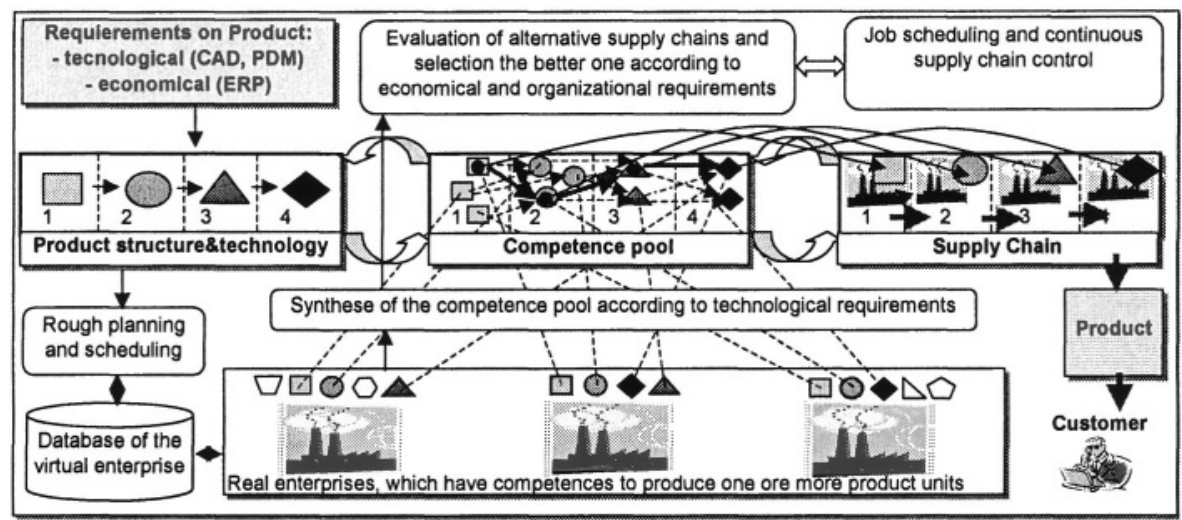

Figure 1- Model of integrated networked supply chain planning

Planning of large-scale processes with complex goals and constraints can hardly be done using a centralized dispatcher and traditional methods focusing on deterministic or stochastic systems. That's why the problem of how to design networked supply chain planning and control with the use of intelligent agents is a subject of increasing interest from both the academic and industrial research communities (Fox, 1994, Rzevski, 1997, Parunak, 1999, Shen, 1999, Rabelo, 2002).

\section{RELATED WORKS}

The modern approaches of VE modeling and structuring are based on small autonomous elements that collaborate which each other for solving of certain tasks in an adjusted application area. The origin of these ideas lies in cellular 
manufacturing systems (Shaw, 1987). In the concept of holonic manufacturing systems (Wang, 2001) corresponding systems are standing out for the balanced proportion of stability and flexibility of its structures. The autonomy of the elements (agents) of this structure covers not only the operative process but also the process of the formation of the structure. The decomposition of a system in autonomous elements (called Holons) forms the cooperative elements. Concept of nonhierarchical, regional production networks is based on small production units, so called competence cells (Teich, 2003). These units possess only few and very specific key competencies. Because of the order-specific selection of those small units, it becomes possible to quickly and flexibly react in case of changing market demands. By the nature these small autonomous elements are very similar to intelligent agents.

Intelligent agents represent a modern approach in Artifical Intelligence and are dealing with software entities which possess competences and resources, can act autonomously, reactive and adaptive, communicate with other agents, are goaloriented, and are using explicit knowledge (Weiss, 2000). In recent years, the concepts of utilization of multi-agent paradigm for supply chain management and virtual enterprises have been strongly developed (Shen, 2001). For such problem areas as modeling supply chain dynamics (Swaminathan, 1998), real-time flexible supply chain structuring (Zeng, 1999), production planning and control in VE (Camarinha-Matos, 1999), distributed dynamic scheduling in manufacturing (Silva, 2002), smart coordination of dynamic supply chains (Rabelo, 2002), decentralized learning in enterprise collaborative systems (Gangli, 2003) the have been proposed adequate models and algorithms based on multi-agent systems (MAS) and modern heuristics.

The deficit of recent researches has been the separative consideration of analysing, modeling, scheduling and control phases in SCM with the use of various methods and models for all these phases, which have not been concerned methodological to each other. The introduction of the planning function can help to develop a methodological framework of virtual enterprise researching.

\section{A GENERAL FORMULATION FOR SUPPLY CHAIN PLANNING IN VIRTUAL ENTERPRISES}

In our approach, the problem of structural-functional synthesis of supply chains is formulated in such a way in order to create a unified basis of supply chain analysing, modelling and control taking into account external influences. The ground of this formulation is the recent results, which have been gained in the theory of structure dynamic control (Sokolov, 2003). Let $B=\left\{B_{i}, i \in M=\{1, \ldots, m\}\right\}$ be a set of participant enterprises; let $\bar{B}=\left\{\bar{B}_{i}, i \in \bar{M}=\{1, \ldots, \bar{m}\}\right\}$ be a set of orders to be planed; $\widetilde{B}=B \cup \bar{B}$ - set of objects for structural-functional synthesis of supply chains in virtual enterprise. Let us introduce a set $C=\left\{C_{\lambda}^{(i)}, \lambda \in \Lambda_{i}, i \in M\right\}$ of requisite competences and a set $\bar{C}=\left\{\bar{C}_{\lambda}^{(l)}, \lambda \in \bar{\Lambda}_{l}, i \in \bar{M}\right\}$ of available competences. Let us also introduce the following 
sets: $D=\left\{D^{(\mathfrak{c})} \cup\left\{D_{\mathbf{x}^{\prime}}^{(l)}\right\}, i, j \in M, \mathfrak{x} \in K_{i}^{(\mathrm{o})}\right\}-$ set of jobs, which can be realized in the VE; $D^{(i)}=\left\{D_{\infty}^{(i)}, æ \in K_{i}^{(o)}=\left\{1, \ldots, s_{i}\right\}\right.$ - set of jobs for the $\bar{B}_{i}$ order; $\left\{D_{i w f \eta_{1}}^{(c, 1)}\right\}-$ set of macro operations, which describe the functioning of the VE $\boldsymbol{B}_{\boldsymbol{i}}$ in the macro state $S_{l w f}$ at the $\eta_{1}$ control loop; $\left\{D_{i w f}^{(0,3)}\right\}$ - set of macro operations, which describe the transition of the VE $B_{i}$ from the current macro state $S_{l w^{\prime} f}$ into the goal state $S_{i w f}$; $\Phi=\left\{\left\{\Phi S_{\pi}^{(i)}\right\} \cup\left\{\Phi N_{\mu}^{(i)}\right\}, i \in M, \pi \in K_{i}^{(\mathrm{p}, 1)}=\left\{1, \ldots, k_{i}^{(\mathrm{p}, 1)}\right\}, \mu \in K_{i}^{(\mathrm{p}, 2)}=\left\{1, \ldots, k_{i}^{(\mathrm{p}, 1)}\right\}\right\}$ - set of resources, which are current available in the VE; $P=\left\{\left\{P_{\left\langle x^{\prime}, \rho>\right.}^{(i)}\right\} \cup\left\{P_{\langle x, \rho\rangle}^{(i, j)}\right\}, i \in M, \mathfrak{x}^{\prime} \in K_{i}^{(0)}, \mathfrak{x} \in K_{<, j\rangle}^{(0)}, \rho \in K_{i}^{(\mathrm{n})}\right\}-$ set of flows (material, informational etc.); $G=\left\{G_{\chi}, \chi \in N S\right\}$ - set of structures, which are being formed while synthesis (topological, technological, technical, organizational, informational etc.)

Let a dynamical alternative multi-graph $G_{\chi}^{\prime}=\left\langle X_{\chi}^{t}, F_{\chi}^{\prime}, Z_{\chi}^{t}\right\rangle$ be brought into consideration to relate the above-named sets and structures, where $\chi$ - type of the structure, $\chi \in N S=\{1,2,3,4,5,6\}, t \in T-$ set of instants of time; $X_{\chi}^{t}=\left\{x_{\chi l}^{t}, l \in L_{\chi}\right\}-$ set of elements of the structure $G_{\chi}^{t}$ (set of knots) at the instant of time $t$; $F_{\chi}^{t}=\left\{f_{\left\langle\chi, l, l^{\prime}\right\rangle}^{t}, l, l^{\prime} \in L_{\chi}\right\} \quad-$ set of arcs $G_{\chi}^{t}$, at the instant of time $t$; $Z_{\chi}^{l}=\left\{f_{\left\langle\chi, l, l^{\prime}\right\rangle}^{t}, l, l^{\prime} \in L_{\chi}\right\}-$ set of parameters which quantify the relations between the elements of the graph (e.g, lead time, costs etc.).

The $G_{\chi}^{t}$ is characterized by macro-structural states and reflections. Let the set of permissible reflections of the graphs $G_{\chi}^{t}$ to each other be denoted as $M_{<\chi, \chi^{\prime}>}^{t}: F_{\chi}^{t} \rightarrow F_{\chi^{\prime}}^{t}$ and composition operations at the instant of time $t$ as $M_{\left\langle x, x^{\prime}\right\rangle}^{\prime}=M_{\left\langle x, x_{1}\right\rangle}^{\prime} \circ M_{\left\langle x_{1}, x_{2}\right\rangle}^{\prime} \circ \ldots \circ M_{\left\langle x^{\prime}, x^{\prime}\right\rangle}^{\prime}$. The macro state of the VE can be defined as $S_{\delta} \subseteq X_{1}^{t} \times X_{2}^{t} \times X_{3}^{t} \times X_{4}^{t} \times X_{5}^{t} \times X_{6}^{t}, \quad \delta=1, \ldots, K_{\Delta}$.

The goal is to find such $\left\langle U^{t}, S_{\delta}^{t_{f}}>\right.$ by the following constraints.

$$
\begin{aligned}
& J_{\theta}\left(X_{\chi}^{t}, \Gamma_{\chi}^{t}, Z_{\chi}^{t}, F_{<\chi, \chi^{\prime}>}^{t}, \Pi_{<\tilde{\delta}, \tilde{\delta}\rangle}^{t}, t \in\left(t_{0}, t_{f}\right]\right) \rightarrow \underset{\left\langle U^{\prime}, S_{\delta}^{S^{\prime \prime}}>\in \Delta_{g}\right.}{\operatorname{extr}},
\end{aligned}
$$

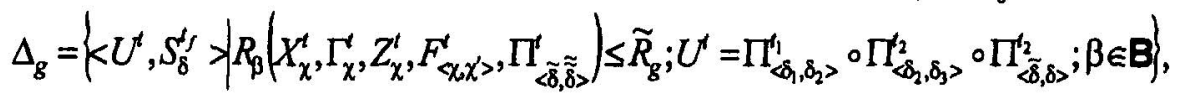

where $U^{\prime}$-control actions for synthesis, $J_{\theta}$-parameters (costs, time, risk etc.) of the VE functioning, $\theta \in \Theta=\{1, \ldots, l\}-$ set of parameter numbers; $\Delta_{\mathbf{g}}-$ set of dynamic alternatives of supply chains; $\mathbf{B}$ - set of process numbers; $\widetilde{R}_{\mathbf{g}}$ - parameters of customer's order; $T=\left(t_{0}, t_{f}\right]-$ interval of time for synthesis. 


\section{AGENT-BASED SUPPLY CHAIN PLANNING}

Effective supply chain planning is based on a common framework of analyzing, modeling and synthesizing of plans. This framework has, at first, to correspond to the characteristic properties of VE. Second, it has to integrate supply chain planning. We propose to extend the utilization of multi-agent approach for the method of complex analysing, modelling and control in VE. Thus MAS can be considered as a complex framework of integrated supply chain planning, because of characteristics corresponding to requirements on SCM in VE (s. table 1). According to these requirements, the agent-oriented approach for VE seems to be more efficient as object oriented one because of taking into account activity and competition.

Table 1 - Requirements on SCM in VE and characteristics features of MAS

\begin{tabular}{|l|l|}
\hline \multicolumn{1}{|c|}{ Requirements on SCM in VE } & \multicolumn{1}{c|}{ Characteristics features of MAS } \\
\hline $\begin{array}{l}\text { Conceptual analyzing and modeling } \\
\text { based on small autonomous elements }\end{array}$ & $\begin{array}{l}\text { Autonomous intelligent agents which } \\
\text { possess competences and resources }\end{array}$ \\
\hline Decentralized planning and control & Decentralized approach \\
\hline $\begin{array}{l}\text { Flexible configuration of supply } \\
\text { chains in distributive manner }\end{array}$ & $\begin{array}{l}\text { Dynamical synthesis of new } \\
\text { structures by agent interactions }\end{array}$ \\
\hline Fast adaptation to market changes & Agents are adaptive \\
\hline Coordinated collaborative actions & Coherency property \\
\hline Complex analyzing of the VE & Ontological analyze \\
\hline $\begin{array}{l}\text { Intelligent integration of } \\
\text { heterogeneous information systems }\end{array}$ & $\begin{array}{l}\text { MAS is a framework of data and } \\
\text { knowledge integration }\end{array}$ \\
\hline $\begin{array}{l}\text { Benefits through real-time } \\
\text { coordination and collaboration }\end{array}$ & $\begin{array}{l}\text { Agents are able to gain benefit by } \\
\text { coordination and collaboration }\end{array}$ \\
\hline
\end{tabular}

The use of MAS facilitates a common representation of objects and concepts through the elaboration of the VE ontology as a unified contract specification system. Moreover, the utilization of MAS for VE framework makes the use of optimisation methods, which are also based on agent principles such as method ACO (Ant Colony Optimisation) more efficient (Teich, Ivanov, 2002).

The MAS functional architecture designed for networked supply chain planning consists of enterprise agents, order agents supply chain agents, which build the functional part, and cooperative knowledge base, a system buffer and information tools, which forms the technical background for information and knowledge management. Enterprise, order and supply chain agents are semantic agents. There are also agents groups of syntactical and morphological levels (Ivanov, Arkhipov, 2003). Building of these agents occurs dynamically by decomposing of the tasks of semantic agents. The technical background consists of cooperative knowledge base, a system buffer (a cooperative blackboard for agent auctions and a cooperative Scoreboard for results evaluation) and program tools for information and knowledge management. A general agent model is shown in the figure 2. 


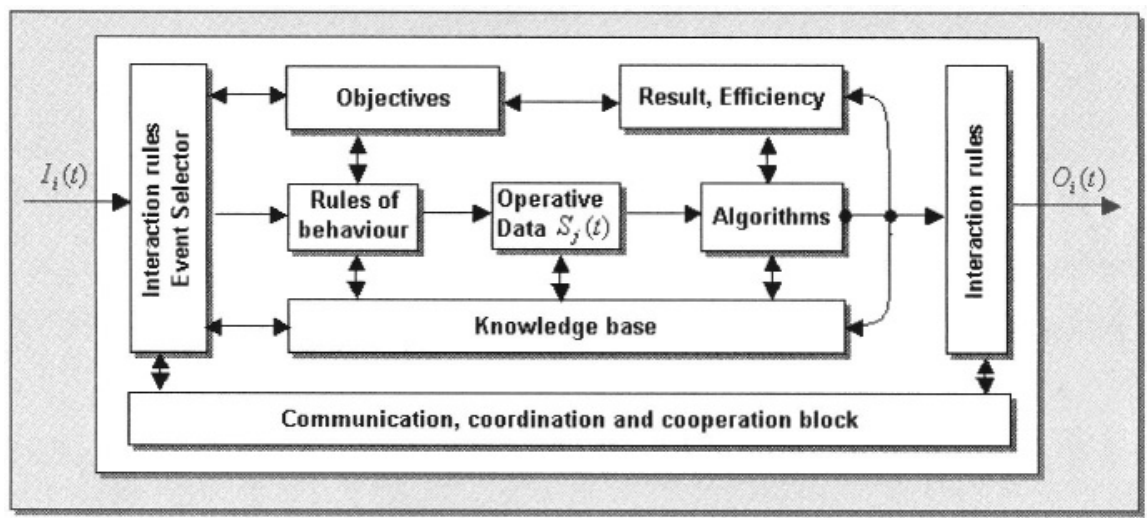

Figure 2 - Agent functional model

An agent is defined by the following set of characteristics.

$S_{j}(t)=$ Set of attributes that characterize its (simulated) state at a given instant of time. State information includes base information about agent's competences (e.g., technological resources) and processing state (e.g., current product inventory, capable resources, production costs etc.).

$D(t)=$ Knowledge base of agent.

$I_{i}(t)=$ Set of incoming messages at agent.

$O_{i}(t)=$ Set of outgoing messages at agent.

$P\left(D, S_{j}, I_{i}, O_{i}\right)=$ A selector function that chooses and sequences a set of incoming messages based on domain knowledge, current state and priorities of agent.

Agent interactions while supply chain planning are described below (s. also Ivanov, Kaeschel, 2003). Enterprise agents (EA) represent network participants. All the agents have permanent access to databases containing general information related to products (CAD, PDM) and operative information related to resources (ERP/SCM/MES). Each agent represents the competences of a real enterprise. The main aim of an EA is to maximize its profit. They aim to gaining the most profitable orders. The EA's offer their competences and compete with each other to get a job or more in the supply chain. The selected EA's communicate with the SCA while producing and transmit the actual production Figures.

Order agents (OA) maintain customers' orders beginning from their incoming up to finishing. Each order will be managed by an OA. The OA coordinates interactions of the first planning level. The main aim of the OA is to synthesize a rough production plan and the competence pool. For this goal the OA uses information about average estimations for order positions from the MAS knowledge base, interacts with an external technological system (PDM, CAD) and with EA's.

Supply chain agents plan and manage supply chains. Each supply chain will be managed by an SCA. The SCA co-ordinates interactions on the second and third planning level as well as on the control level. The main aim of the SCA is to synthesize supply chain structure of the various alternatives of the competence pool, to schedule and to control the supply chain. The SCA evaluates the alternative 
supply chains of the competence pool and select the better one. The enterprises for each technological operation of each BOM position will be selected using information concerning the current shop flow situation (e.g. from MES manufacturing execution systems) as well as information about organizational constraints - soft-facts (e.g. reliability, trust etc.). The better supply chain is resulting from auctions between appropriate partners of the competence pool. Then the supply chain is scheduled. While verifying of this detailed plan the SCA also interact with the EA's and the OA. The third planning level is meant for detailed scheduling within the synthesized supply chain. After production starting the continuos control phase takes place and lasts until the order is finished.

Valuable knowledge obtained by agents while carrying out the order is saved in their knowledge base and can be used in new actions. The saved parameters are also available after job finishing and may be used to solve possible conflicts. Moreover, cooperative Scoreboard of intelligent agents makes it possible to control and to change cooperative partner processes especially efficiently. The other advantage of agent-based technology is that it makes the use of agent-based optimisation algorithms of scheduling such as ACO more efficient (Ivanov, Kaeschel, 2003).

\section{CURRENT RESEARCH STATE AND FURTHER PLAN}

Our main research objective is to develop a system of supply chain management in $\mathrm{VE}$ and to evaluate the results from simulation of case examples on a real production network existing in Saint Petersburg and Moskau. The ground of this research will be the recent mathematical results, which were gained in the theory of structure dynamic control and facilitate the dynamic consideration of SCM in VE. Some of these results are realized as software SDC (Structure Dynamic Control) at Saint Petersburg Institute of Informatics and Automation as part of the international partner project of the EOARD №1992p “The Optimal Structure Reconfiguration in a Complex Technical System".

Software SDC facilitates real-time planning and monitoring of the processes and states in complex systems. Current state of the SDC facilitates synthesizing of supply chains in case of simply ontology (if there is a centre, which co-ordinates the activities in the VE and distributes the jobs to the enterprises in accordance to the available resources). The further direction of our researches is to provide the software with the agent oriented technologies so as to integrate it in the general information on space of the VE (Ivanov, Arkhipov, 2003). Introduction of intelligent agents as additional program modules can help to realize the ontology, when the enterprises compete and act non-cooperatively. 


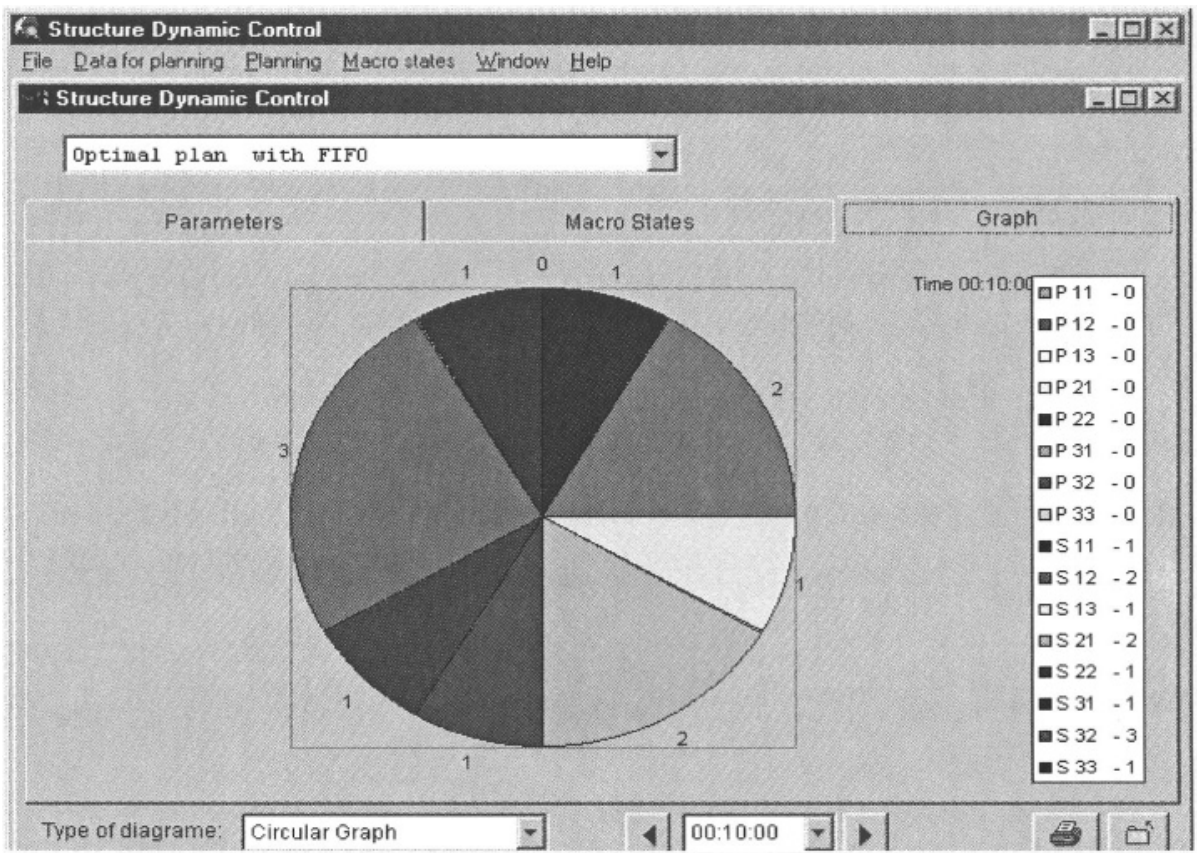

Figure 3. Software SDC (Structure Dynamic Control)

\section{CONCLUSION}

Efficient supply chain planning in virtual enterprises is based on a common framework of analyzing, modeling and synthesizing of plans. In this paper, we presented methodological basis of such a framework with the use of multi-agent paradigm. We proposed to extend the utilization of multi-agent approach for the method of complex analyzing and modeling in virtual enterprises. For its realization we elaborated the functional agent model and MAS architecture for intelligent support of collaborative processes in virtual enterprises. A general formulation of dynamic supply chain planning in virtual enterprises was given. We plan to enrich our concept through extension of models of dynamical structuring in virtual enterprises. We are currently working on integrating planning, scheduling and control models as well as on scheduling optimization algorithms. We are also working on adaptation and risk models. The goal of our researches will be to design the unified methodological and technical basis of supply chain analysing, modelling and control and to evaluate the results from simulation of case examples on a real production network existing in Russia. 


\section{REFERENCES}

1. Camarinha-Matos LM, Silva VS, Rabelo RJ. Production Planning and Control in a Virtual Enterprise. PRO-VE 1999: Infrastructures for Virtual Enterprises (L.M. Camarinha-Matos, H. Afsarmanesh- Eds.), Kluwer Academic Publishers, ISBN 0-7923-8639-6, pp. 219-232, Oct 99, Porto, Portugal.

2. Fox M. ISIS: A retrospective. Intelligent Scheduling, 1: 3-28, Morgan Kaufmann, 1994.

3. Gangli Q, Jiaben Y. Temporal Decentralized Co-Evolutionary Learning Agent Model for Enterprises Collaborative System. In Proceedings of the 13th International Conference on flexible Automation \& Intelligent Manufacturing, Tampa, USA, 2003, 1:679-687.

4. Ivanov DA, Arkhipov AV. A Conception of a Model-Based System of Operative Order Control in Cooperative Production Networks with the Use of Intelligent Agents. In Proceedings of the 3rd international conference on Research and Development in Mechanical Industry, RaDMI 2003, Herceg Novi (Montenegro Adriatic), Yugoslavia, 2003: 237-246.

5. Ivanov DA, Käschel J. Vernetzte Planung und kontinuierliche Lieferkettenoptimierung. PPSManagement, 2003,4:29-32.

6. Ivanov, Dmitry. Virtual Enterprises and Supply Chains: a Complex Approach to Organization and Operative Control in New Forms of Industrial Co-Operaion. St. Petersburg: SPbGUEF, 2003.

7. Kaihara T. Supply Chain Management based on Market Mechanism in Virtual Enterprise, Infrastructures for Virtual Enterprises, Kluwer Academic Publishers, Boston, 1999: 399-408.

8. Multiagent Systems: a Modern Approach to Distributed Artificial Intelligence (ed. by G.Weiss). The MIT Press, Cambridge, Massachusetts, London, 2000.

9. Parunak H. Industrial and Practical Applications of DAI, 9: 377-421. MIT Press, 1999.

10. Rabelo RJ, Klen AAP, Klen ER: A Multi-Agent System for Smart Coordination of Dynamic Supply Chains. PRO-VE 2002: 379-387.

11. Rzevski G. A framework for designing intelligent manufacturing systems. Computers in Industry, 1997, 34:211-219.

12. Shaw MJP. Dynamic scheduling in cellular manufacturing systems: a framework for networked decision making. International Journal of Production Research, 1987, 7: 83-94.

13. Shen, Weiming et. al. Multi-Agent Systems for Concurrent Intelligent Design and Manufacturing. Taylor \& Francis Group, 2001.

14. Shen W., Norrie DH. Implementing internet enabled virtual enterprises using collaborative agents. In Infrastructures for Virtual Enterprises, Camarinha-Matos, L.M., 1999: 343-352.

15. Silva CA, Runkler T, Sousa JM, Palm R. Optimization of logistic processes using ant colonies. In: Proceedings of Agent-Based Simulation, 3:143-148.

16. Sokolov B.V. Optimal Structure Reconfiguration in a Complex Technical Systems(CTS): principles, models, methods and algorithms for the CTS Structure Dynamics Control // VI ISTC Scientific Advisory Committee Seminar "Science and Computing". - Moscow, 2003, pp. 30-31.

17. Swaminathan JM, Smith SF, Sadeh NM. Modeling Supply Chain Dynamics: A Multiagent Approach. Decision Science, 1998, 29(3): 607-632.

18. Teich T. Extended Value Chain Management (EVCM). GUC-Verlag, 2003.

19. Teich T, Ivanov DA: A cooperative learning approach to SCM in virtual enterprises using heutistic algorithms and multi-agent systems. In Proceedings of the 13th international DAAAM symposium "Intelligent Manufacturing \& Automation: Learning from the Nature", Austria, Vienna, 2002, 563564.

20. Wang L, Norrie DH. Process Planning and Control in a Holonic Manufacturing Environment. Journal of Applied Systems Studies, 2001, 2(1): 106-126.

21. Zeng DD, Sycara K.P. Agent-Facilitated Real-Time Flexible Supply Chain Structuring. In Proceedings of Workshop on Agent Based Decision-Support for Managing the Internet-Enabled Supply-Chain, Seattle, Washington, 1999, 21-28. 